for IPV was significantly associated with annual income and the type of help received from the EAP. Approximately $71 \%$ of EAPs users reported that their work performance improved after contacting their EAP. The most common barrier to women seeking help from the EAP was concerns with confidentiality. EAPs appear to be a useful resource for helping women impacted by IPV. Future efforts to increase satisfaction and enhance the role of EAPs in addressing IPV should address confidentially and strengthen how the delivery of IPV-related assistance occurs.

\title{
0851 UNDERSTANDING WOMEN'S EXPERIENCES WITH EMPLOYEE ASSISTANCE PROGRAMS FOR ASSISTANCE WITH INTIMATE PARTNER VIOLENCE
}

K M Pollack*, C Cumminskey, M Solomon, K Krotki, W Austin, J A Grisso Correspondence: School of Public Health, Johns Hopkins University Bloomberg, 624 N. Broadway, Room 557 Baltimore, MD 21205, USA

10.1136/ip.2010.029215.851

Intimate partner violence (IPV) is a serious, preventable global public health problem with significant impacts on work. Limited research exists on the scope of services and programs that Employee Assistance Programs (EAPs) offer to address IPV and the experiences of IPV victims when they interact with EAPs. To address this gap, we used a web panel to identify women to complete a survey on their experiences with the EAP for IPV. Data were collected from a nationally representative sample of employed women, 18 years of age and older, living in the U.S. A total of 1765 employed women were surveyed: 760 women contacted their EAP about their experiences with IPV and 1005 women did not contact their EAP about these experiences. Most women (91\%) contacted the EAP in need of counselling, especially for mental health treatment and legal services. Most women (63\%) were satisfied with the EAP services they received. Multivariate logistic regression analysis showed that satisfaction with EAP services 